\title{
Celiac disease, still an uncommon problem in Tamilians?
}

Celiac disease (CD) is the most common cause for malabsorption in Western nations with an estimated prevalence of one case per $99-250$ population. ${ }^{1,2}$ A similar high prevalence of one in 310 has been reported from northern India ${ }^{3}$ thus expanding the "global village of CD". This scenario is not seen in Chennai, South India, a predominantly riceconsuming region where only three cases of $\mathrm{CD}$ have been reported in literature. ${ }^{4}$ This variation is probably due to the different genetic background and antigenic exposure. ${ }^{5}$ Since 2005, no published data on CD are available from South India.

We examined the records of children attending the Gastroenterology Clinic of our hospital, which is a tertiary care pediatric hospital, between March 2006 and February 2009 with chronic diarrhea, unexplained growth retardation with or without anemia, and normal serum $\operatorname{IgA}$, and in whom $\operatorname{IgA}$ anti-tissue transglutaminase antibody (IgA anti-tTG) was performed. During this period, 98 children (19 from North India) reported with chronic diarrhea and 27 required admission. Seventy-five children (76\%) were tested for IgA anti-tTG and chronic diarrhea was the indication in $72(96 \%)$ children. IgA antibodies against tTG was detected using recombinant human tTG constituted with gliadin-specific peptide as the antigen (Aeskulisa TTG-A; Aesku Diagnostics, Germany) and values $>15$ U/L were interpreted as positive.

IgA anti-tTG was positive in 15 children (11 boys) inclusive of the three reported earlier. The mean IgA anti-tTG titer was $61.24 \mathrm{U} / \mathrm{L}$. These children were aged between 15 months and 10 years (median 4.5 years). Ten of them were from northern India, one was from Kerala, two from Andhra Pradesh and two from Tamil Nadu. Duodenal histology using modified Marsh criteria was analyzed in these 15 children. All of them had variable forms of villous atrophy, crypt hyperplasia and increased intra epithelial lymphocytes, and responded to gluten-free diet.

Sixty children were anti-tTG negative; $52(87 \%)$ of them hailed from Tamil Nadu. The other identifiable causes of chronic diarrhea were lactose intolerance and cow's milk protein intolerance in five each, HIV, tuberculosis, cryptosporidiosis, cystic fibrosis and factitious diarrhea in two each, acrodermatitis enteropathica, chronic pancreatitis and intestinal lymphangectasia in three each, Crohn's disease, desquamative enterocolitis, strongyloidiasis and possible sucrase-isomaltase deficiency in one each, while in the rest the cause was not identified.

In developing countries like India, several conditions may result in villous atrophy and therefore serological tests are necessary in addition to the modified ESPGHAN criteria for the diagnosis of CD. IgA anti-tTG has been universally recommended as a serological marker for $\mathrm{CD}$ because of its high sensitivity and specificity (74-100\%) and its diagnostic utility has been recently documented in children from North India. ${ }^{6}$ This study highlights that CD is probably uncommon in Tamilians or there is still a lack of recognition or enthusiasim in reporting.

$$
\begin{array}{r}
\text { R. Ganesh · N. Suresh · M. Sathiyasekaran } \\
\text { Kanchi Kamakoti CHILDS Trust Hospital, } \\
\text { Chennai } 600 \text { 034, India } \\
\text { R. Ganesh ( } \bowtie) \\
\text { E-mail: ganeped79@rediffmail.com }
\end{array}
$$

\section{References}

1. Not T, Horvath K, Hill ID, et al. Celiac disease risk in the USA: high prevalence of antiendomysium antibodies in healthy blood donors. Scand J Gastroenterol 1998;33:494-8.

2. Maki M, Mustalahti K, Kokkonen J, et al. Prevalence of celiac disease among children in Finland. $N$ Engl $J$ Med 2003;348:2517-24.

3. Sood A, Midha V, Sood N, Avasthi G, Seghal A. Prevalence of celiac disease among school children in Punjab, North India. J Gastroenterol Hepatol 2006;21:1622-5.

4. Sathiyasekaran M, Shivbalan S. Celiac disease in Chennai. Indian Pediatr 2005;42:1171-2.

5. Yachha SK, Poddar U. Celiac Disease in India. Indian J Gastroenterol 2007;26:230-7.

6. Yachha SK, Aggarwal R, Srinivas S, Srivastava A, Somani SK, Itha S. Antibody testing in Indian children with celiac disease. Indian J Gastroenterol 2006;25:133-5. 